\title{
Manifestaciones de los Profesores de Matemáticas sobre sus Prácticas de Evaluación de la Resolución de Problemas
}

\section{Manifestations of Mathematics Professors on their Practices of Problem \\ Solving Assessment}

\author{
Janeth A. Cárdenas Lizarazo ${ }^{*}$ \\ Lorenzo J. Blanco Nieto ${ }^{* *}$ \\ Eloísa Guerrero Barona ${ }^{* * *}$ \\ Ana Caballero Carrasco ${ }^{* * * *}$
}

\begin{abstract}
Resumen
La evaluación es uno de los organizadores del currículo que debe formar parte del proceso de enseñanza y aprendizaje, además a través de ella se constata la adquisición de aprendizajes. Conjuntamente, entendemos que resolver problemas es un objetivo de aprendizaje, tal y como se refleja en las propuestas curriculares, por lo que éste se convierte en objeto de evaluación. En nuestra investigación elaboramos un cuestionario sobre la evaluación de la resolución de problemas a partir de las demandas que se hacen en el currículo nacional de matemáticas, y lo aplicamos a 211 profesores de matemáticas de secundaria de Bogotá (Colombia), en busca de analizar los criterios que los profesores dicen considerar al evaluar éste contenido y el nivel de importancia, o de acuerdo, que le otorgan a evaluar cada uno de esos aspectos. Como resultados, encontramos que entre nuestros informantes aunque consideran prioritarias las demandas curriculares en cuanto a la enseñanza y el aprendizaje de las estrategias heurísticas, su evaluación se centra en el desarrollo de procedimientos meramente mecánicos. Igualmente, destacamos, que aunque se reconoce la importancia de la evaluación de los aspectos afectivos, su evaluación es notablemente menos considerada. Esta investigación forma parte de un proyecto más amplio desarrollado entre el Departamento de Didáctica de las Ciencias Experimentales y de las Matemáticas y el Departamento de Psicología y Antropología de la Universidad de Extremadura.
\end{abstract}

Palabras-clave: Resolución de Problemas. Matemáticas. Secundaria. Evaluación. Profesores de Secundaria.

\begin{abstract}
Assessments are one of the organizers of the curriculum and should be part of the teaching and learning, as well as through the acquisition of learning. Together, we understand that problem solving is a learning objective, as

\footnotetext{
"Doctora en Investigación de la Enseñanza-Aprendizaje de las Ciencias Experimentales, Sociales y de las Matemáticas por la Universidad de Extremadura (UEx). Profesor Ayudante Doctor de la Universidad de Zaragoza (Unizar), Zaragoza, España. Dirección postal: Calle Pedro Cerbuna, C.P. 50009, Zaragoza, España. Email: jacarliz@unizar.es

** Doctor en Pedagogía por la Universidad de Sevilla (US). Catedrático de Didáctica de las Matemáticas en la Universidad de Extremadura, Badajoz, Extremadura, España. Dirección postal: Avda. de Elvas s/n, C.P. 06071, Badajoz, España.E-mail: lblanco@unex.es

*** Doctora en Psicología por la Universidad de Extremadura (UEx). Profesor Titular de Universidad en la Universidad de Extremadura, Badajoz, Extremadura, España. Dirección postal: Avda. de Elvas s/n, C.P. 06071, Badajoz, Extremadura, España. E-mail: eloisa@unex.es

**** Doctora en Investigación de la Enseñanza-Aprendizaje de las Ciencias Experimentales, Sociales y de las Matemáticas por la Universidad de Extremadura (UEx). Profesor Ayudante Doctor de la Universidad de la Universidad de Extremadura, Badajoz, Extremadura, España. Dirección postal: Avda. de Elvas s/n, C.P. 06071, Badajoz, Extremadura, España. E-mail: acabcar@unex.es
} 
reflected in the curriculum proposals, so that it becomes subject to evaluation. In our research we developed a questionnaire assessing problem solving from the demands made in the national curriculum of mathematics and applied it to 211 high school mathematics teachers in Bogota (Colombia), seeking to analyze the criteria that teachers consider when evaluating this content and level of importance or agreement, which is used to evaluate each one of these aspects. As a result, we find that among our informants, but as priorities the curricular demands on teaching and learning heuristics, evaluation focuses on the development of purely mechanical means. Also we include, although the importance of assessing affective is recognized, its evaluation is considered less markedly. This research is part of a larger project in the Department of Didactics of Experimental Sciences and Mathematics at the University of Extremadura.

Keywords: Problem solving. Math. Secondary. Evaluation. High school teachers

\section{La evaluación en matemáticas}

Nadie discute que la evaluación debe ser una parte integrada dentro del proceso de Enseñanza y Aprendizaje (E/A), que debe ayudar a los profesores a comprender mejor lo que los estudiantes saben y aprenden y a proporcionarles una información importante para tomar decisiones significativas sobre su programación y trabajo en el aula.

Al respecto, en las últimas décadas son numerosos los países que han desarrollado diferentes cambios en las propuestas curriculares en busca de integrar la evaluación al proceso de E/A. Sin embargo, la comunidad educativa considera que la evaluación sigue estando desvinculada en espacios y tiempos (CASTRO; MARTÍNEZ; FIGUEROA, 2009; GOÑI, 2008). Además, entre los profesores existe una opinión generalizada de que los criterios e instrumentos de evaluación utilizados en el aula de matemáticas en secundaria han evolucionado muy poco (CÁRDENAS, 2014). Por lo que se evidencia el desarrollo de actuaciones no acordes con las propuestas curriculares (GODOY, 2013; ÁLVAREZ; BLANCO, 2014).

En acuerdo con Goñi (2008), consideramos el currículo como fuente de organización, secuenciación y preparación de las clases, puesto que detalla indicaciones sobre los aprendizajes que deben conseguir los estudiantes al finalizar los diferentes niveles educativos. A su vez, asumimos que el profesor selecciona, implícita o explícitamente, los elementos del currículo y decide cómo desarrollarlos en el aula en aras a conseguir una enseñanza más eficaz. Así, será él quien determine las intenciones que guían el currículo implementado, tomando marcos de referencia semejantes o criterios ampliamente compartidos desde su experiencia o su formación, tal y como lo afirma Goñi (2011). De esta manera, la evaluación refleja las concepciones de los profesores sobre las matemáticas escolares y el papel que la evaluación juega en el proceso de E/A (CASTRO; MARTÍNEZ; FIGUEROA, 2009), de forma que su análisis ayudaría a identificar estas variables (BROWN; REMESAL, 2012). 
Para Acevedo et al. (2005) el profesor, a través de la evaluación, dota de importancia el contenido e identifica los elementos importantes del proceso de E/A. Álvarez y Blanco (2014) indican que el profesor realiza un mayor énfasis en el contenido que evalúa en el aula con el fin de que los resultados que se obtengan en la evaluación sean óptimos. Además, el profesor selecciona los criterios e instrumentos de evaluación pero, aunque son diversas las fuentes para diseñar los instrumentos de la evaluación, repite los métodos tradicionales que vivió como discente en su práctica docente.

De esta forma, las decisiones que se toman sobre la evaluación repercuten de manera decisiva en el aprendizaje, ya que los estudiantes centran su atención y esfuerzos en el contenido que se les evaluará en busca de ser aprobados. En este sentido, Harlen (2012) indica que la evaluación determina el qué, el cómo y el cuándo los alumnos estudian/aprenden, ya que ellos escogen sus formas de estudiar/aprender para acomodarse a la forma en que serán evaluados. Consecuentemente, podríamos asumir que los objetivos que no se evalúan difícilmente serán alcanzados.

Ello nos sugiere profundizar en las concepciones y prácticas del profesorado sobre la evaluación en matemáticas, tomando como fuente las indicaciones dadas en el currículo sobre un contenido específico. En nuestro caso la resolución de problemas.

\subsection{La resolución de problema en el currículo de matemáticas y su evaluación}

La presencia de la resolución de problemas (RP) se refuerza en los currículos a partir de los 80’s y siguientes, y en su investigación en la educación matemática sigue vigente. Para Castro (2008), la presencia e importancia de la RP se ha mantenido e incluso acrecentado en las propuestas curriculares, tanto nacionales como internacionales, aunque su repercusión en las aulas no ha sido acorde con lo deseado en los objetivos de los currículos. Puig (2008) señala que esta presencia se acompañó de una tensión mal resuelta entre la doble consideración de la RP como contenido y como metodología, lo cual perjudicó su tratamiento como contenido en los diseños curriculares y diluyó su presencia en la práctica en ambos sentidos.

Por otra parte, encontramos que los intentos realizados para enseñar a los alumnos estrategias generales de RP no han tenido éxito (CASTRO, 2008; SANTOS-TRIGO, 2008), y que los bajos resultados que se obtienen en los informes internacionales, como el informe PISA 2012 (OECD, 2014) y el estudio realizado por la International Association for the Evaluation of Educational Achievement (IEA) a través de la prueba denominada TIMSS 
(MULLIS et al., 2012), dejan en entredicho el aprendizaje de la RP como contenido. Así como la falta de atención sobre el aprendizaje de estrategias heurísticas para la RP en los libros de texto (PINO; BLANCO, 2008; SCHOENFELD, 2007).

Al analizar el currículo colombiano de matemáticas (MEN, 1998, 2006) encontramos que en él se reconoce la RP como contenido y como metodología; y, en busca de comprender el significado específico de la RP como contenido y describir cuáles son las indicaciones que se establecen en el currículo para favorecer el aprender a resolver problemas en los estudiantes realizamos un análisis de contenido sobre éste. Para ello, dividimos el texto en unidades de análisis (UA), definidas como palabras, frases o conjunto de frases literales del texto analizado y que tienen significado en relación a los objetivos que se pretenden (BLANCO; BARRANTES, 2003). En nuestro caso las unidades de análisis son un fragmento del texto, de unidad variable, que nos muestra una idea clara en relación a la RP de matemáticas (Cuadro 1).

\begin{tabular}{|l|l|}
\hline \multicolumn{1}{|c|}{ Unidad de Análisis } & \multicolumn{1}{c|}{ Significado } \\
\hline $\begin{array}{l}\text { "Para resolver un problema es necesario, a partir de la } \\
\text { comprensión inicial..." (MEN, 1998, p. 90). }\end{array}$ & $\begin{array}{l}\text { Es evidente que el currículo le da importancia a la } \\
\text { compresión de la situación planteada para diseñar } \\
\text { algún proceso de resolución. }\end{array}$ \\
\hline $\begin{array}{l}\text { "El pensamiento numérico implica reconocer que con } \\
\text { frecuencia existen diferentes estrategias de solución } \\
\text { para un problema dado" (MEN, 1998, p.36). }\end{array}$ & $\begin{array}{l}\text { De modo similar se encuentran otras UA que refieren } \\
\text { a los otros pensamientos, por lo que deducimos que en } \\
\text { el currículo se manifiesta que se deben considerar } \\
\text { diferentes estrategias de resolución y no un único } \\
\text { procedimiento. }\end{array}$ \\
\hline $\begin{array}{l}\text { "La evaluación formativa ha de poner énfasis en la } \\
\text { valoración permanente de las distintas actuaciones de } \\
\text { los estudiantes cuando interpretan y tratan situaciones } \\
\text { matemáticas y a partir de ellas formulan y solucionan } \\
\text { problemas" (MEN, 2006, p. 75). }\end{array}$ & $\begin{array}{l}\text { Interpretar, formular y solucionar problemas son } \\
\text { acciones que se deben evaluar en matemáticas. }\end{array}$ \\
\hline
\end{tabular}

Cuadro 1 - Ejemplo de UA extraídas del (MEN, 1998; 2006) y el significado que le hemos dado

El conjunto de las UA extraídas nos llevó a considerar siete categorías, que se formulan a partir de acciones específicas sobre la RP que aparecen en el currículo y expresan ideas semejantes sobre: i. El formular o plantear problemas; ii. El modelo general para la RP: analizar y comprender el problema; diseñar estrategias; ejecutar las estrategias; revisar el problema, el resultado y tomar de decisiones; iii. Los aspectos del dominio afectivo presentes en la RP; iv. El uso de la tecnología de la información y de la comunicación en la $R P$; v. Las fuentes de situaciones y datos para plantear y resolver problemas; vi. Las representaciones matemáticas, su lenguaje y comunicación; vii. Su evaluación.

\section{2 ¿Qué evaluar sobre la resolución de problemas?}


El análisis del currículo indica que la evaluación debe determinar la capacidad que tiene el alumno para realizar todos los aspectos de la RP. Las categorías del $i$ al vi muestran los diferentes contenidos relacionados con el aprender a resolver problemas y, como tal, deben ser objeto de evaluación si se asume que ésta forma parte del proceso de E/A. SantosTrigo (2007, p. 171) dice que "es importante diseñar actividades adecuadas que capturen información de los diferentes momentos identificados en el modelo [de RP]", y anima a hacer que los estudiantes verbalicen sus pensamientos, dudas, y conjeturas. En definitiva, que expliquen lo que ha pasado por su mente y lo que han ido haciendo, para verificar y evaluar si el alumnado trata de resolver el problema de forma lógica y reflexiva.

La estructura curricular de matemáticas en Colombia se basa en los procesos generales (formular y resolver problemas; modelar procesos y fenómenos de la realidad; comunicar; razonar; y, formular, comparar y ejercitar procedimientos algorítmicos), los conocimientos matemáticos y los contextos de aprendizaje en busca de potenciar las competencias matemáticas (MEN, 1998; 2006), reconociendo relaciones e interacciones múltiples entre estos componentes. Específicamente, se considera que la formulación y la resolución de problemas involucra a todos los demás procesos generales con distinta intensidad en sus diferentes momentos (MEN, 2006). Además se menciona que "a través de los procesos generales, se explicita lo que significa ser matemáticamente competente" (MEN, 2006, p. 51), para ello se condensa el modelo de RP, se mencionan algunos heurísticos y se comenta el papel fundamental de la comunicación en la RP.

También se indica que en la evaluación se ha de poner énfasis en la valoración permanente de las distintas actuaciones de los estudiantes cuando interpretan y tratan situaciones matemáticas y formulan y solucionan problemas a partir de ellas (MEN, 2006).

Consecuentemente, entre otras cuestiones, en la evaluación sobre la RP deberíamos comprobar que el alumno es capaz de recoger y registrar información que se pueda cuantificar, formular problemas a partir de una información general y utilizar algunos recursos sencillos de representación gráfica (tablas, diagramas, ecuaciones etc.) donde entiende y comunica la información ahí expresada. Igualmente, habría que observar si comprueba o no los datos, si compara los resultados obtenidos con la situación original para ver lo razonable de los mismos o si realiza o no algún tipo de generalización. La capacidad para transferir conocimiento (conceptual o procedimental) puede observarse al final del proceso de resolución al plantear a los alumnos, que a partir del problema resuelto, formulen nuevos problemas en diferentes contextos.

Ligado al procedimiento seguido en la RP, la evaluación debe tener en cuenta las 
creencias y la actitud personal del resolutor ante la RP. Así, el interés por la actividad, la autoconfianza y la valoración que hacen de ellos mismos como resolutores, las expectativas de éxito y autoeficacia, la ansiedad con que se enfrentan a los problemas y la perseverancia ante intentos fallidos son algunos factores que también hay que considerar en la evaluación.

Giménez (1997) en el registro de valoración sobre situaciones de RP establece cinco categorías de evaluación con sus respectivas subcategorías: 1. interpretación (criterios, identificación, reconocimiento, reflexión y crítica); 2. organización (estructuración, control de variables, eficiencia, manipulación y generación de datos y tratamiento de la información); 3. simbolización (análisis, representaciones y convencionalismos y uso correcto de instrumentos); 4. formulación de hipótesis (modelización, establecimiento de relaciones, demostración y justificación y análisis); y 5. comunicación (representación, formulación de soluciones, intervención escrita, intervención oral e intervención grupal).

Por nuestra parte, asumiendo estas aportaciones, consideramos que la evaluación debería realizarse de una manera sistemática y continua para permitir conocer el avance de los alumnos sobre la RP y que éstos lleguen a adquirir confianza y capacidad para esta tarea en contextos diversos, incidiendo en sus procesos metacognitivos y afectivos. Consideramos que una de las razones por las que "los alumnos [...] tienden a aprenderse de memoria los problemas de matemáticas hechos en clase como estrategia metacognitiva" (HIDALGO et al., 2013, p. 230), sea la falta de atención a estos factores.

\section{Problema de investigación}

Los apartados anteriores nos indican la diversidad de aspectos que pueden ser tratados en relación a la RP en matemáticas y que, por tanto, debieran ser considerados en la evaluación. La incidencia de la evaluación en los procesos de E/A, el hecho de que el profesorado siga evaluando de manera muy tradicional (ÁLVAREZ; BLANCO 2014; GODOY, 2013), al igual que las dificultades que se presentan en las correcciones de exámenes (GAIRÍN; MUÑOZ; OLLER, 2012; MENGUAL; GORGORIÓ; ALBARRACÍN, 2013), nos sugiere profundizar en los conocimientos y concepciones de los profesores de matemáticas y analizar su práctica docente en relación a la evaluación sobre la RP de matemáticas. En este sentido, hemos desarrollado un proyecto de investigación, entre los Departamentos de Didáctica de las Ciencias Experimentales y de las Matemáticas y el de Psicología y Antropología de la Universidad de Extremadura, en el que empleamos diferentes instrumentos para indagar sobre la evaluación de la RP. 
En este artículo presentamos algunos resultados obtenidos de un cuestionario de elaboración propia, denominado Cuestionario Evaluación de la Resolución de ProblemasCERP, el cual fue construido a partir de las UA extraídas del currículo. Con él se recoge información acerca de las manifestaciones que hacen los profesores sobre qué evalúan de la $\mathrm{RP}$, cuáles son los aspectos que tienen en cuenta al planificar su evaluación y el nivel de importancia (o de acuerdo) que consideran se debiera dar a la evaluación de dichos aspectos. Además se identifican las manifestaciones que hacen sobre la enseñanza de la RP.

El conocer los aspectos de la RP que los profesores manifiestan relevantes en la enseñanza junto al nivel de importancia que le otorgan (independientemente de si lo evalúa o no) y su contraste con lo que manifiestan evaluar, nos permitirá tener un conocimiento más claro acerca de las concepciones y prácticas en relación al tema que nos ocupa. Ello aportará ideas y sugerencias para diseñar programas de formación de profesores que permitan avanzar en la evaluación en matemáticas de acuerdo a las propuestas curriculares.

\section{Población de estudio, instrumento y metodología de análisis}

\subsection{Población}

Hemos trabajado con una población de 211 profesores de matemáticas de secundaria y bachillerato de los colegios públicos de Bogotá (Colombia), los cuales fueron seleccionados bajo un muestreo aleatorio estratificado. Sus edades varían entre los 24 y los 64 años, siendo 42 años la edad promedio, y su experiencia laboral promedio es de 19 años.

\subsection{Instrumento}

El CERP es un cuestionario de elaboración propia, diseñado a partir del análisis de contenido del currículo de matemáticas de Colombia y diferentes publicaciones sobre la RP en matemáticas. El CERP fue validado entre los años 2011-2012 (CÁDERNAS, 2014), consta de 93 ítems, distribuidos en tres bloques de preguntas:

- Primer bloque (29 ítems): se centra en qué se evalúa en la RP, ya sean acciones para la RP o características de los resolutores (Figura 1);

- Segundo bloque (28 ítems): indaga sobre los aspectos que se pueden considerar al planificar la evaluación de la RP, tales como los problemas planteados, el tipo de 
actividad a desarrollar y las dificultades que pueden presentar los estudiantes (Figura 2);

- Tercer bloque (28 ítems): inquiere sobre los aspectos en los cuales los profesores centran su atención al enseñar la RP (Figura 3).

\begin{tabular}{|c|c|c|c|c|c|c|c|}
\hline \multirow{2}{*}{\multicolumn{2}{|c|}{ ¿Evalúo? }} & & \multicolumn{5}{|c|}{ Nivel de Importancia } \\
\hline & & & \multirow{2}{*}{$\begin{array}{r}\text { Nada } \\
0 \\
\end{array}$} & \multicolumn{2}{|l|}{ Poco } & \multicolumn{2}{|c|}{ Mucho } \\
\hline $\mathrm{Si}$ & No & El distinguir y separar las partes de un problema hasta comprenderlo. & & 1 & 2 & 2 & 4 \\
\hline $\mathrm{Si}$ & No & $\begin{array}{l}\text { E1 hacer uso de diversos lenguajes para expresar ideas matemáticas pertinentes a } \\
\text { partir de situaciones problema. }\end{array}$ & 0 & 1 & 2 & 3 & 4 \\
\hline $\mathrm{Si}$ & No & E1 seleccionar recursos materiales apropiados para resolver problemas. & 0 & 1 & 2 & 3 & 4 \\
\hline $\mathrm{Si}$ & No & La visión que cada estudiante tiene de símismo en la resolución de problemas & 0 & 1 & 2 & 3 & 4 \\
\hline
\end{tabular}

Figura 1 - Ejemplo de ítems del primer bloque de preguntas: qué se evalúa en la RP.
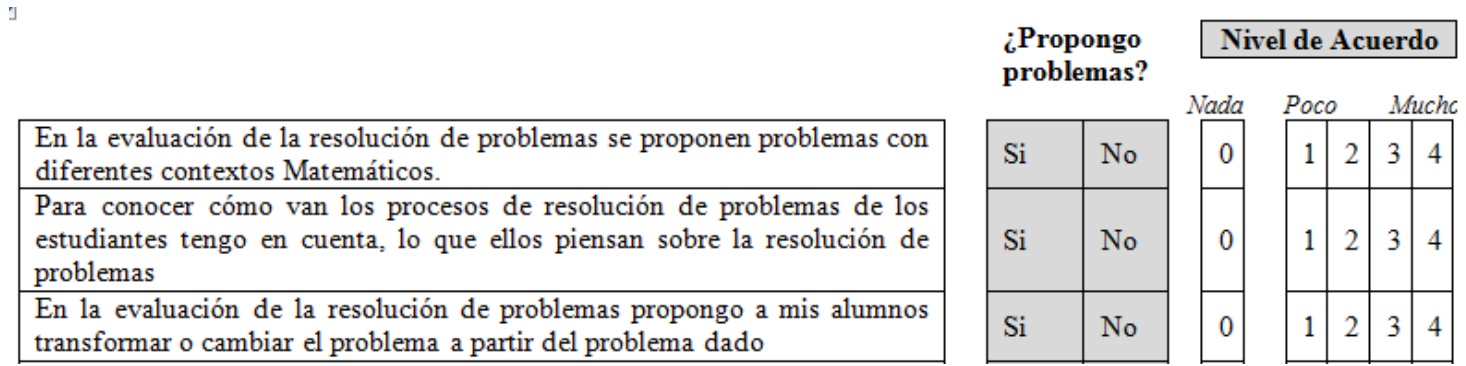

Figura 2 - Ejemplos de ítems del segundo bloque de preguntas: qué planifican en torno a la evaluación de RP.

\begin{tabular}{l} 
Doy importancia a que mis estudiantes al resolver \\
problemas obtengan el resultado exacto \\
\hline Cuando miro cómo resuelve un alumno un problema, doy \\
importancia a que desde el principio vaya por el buen \\
camino \\
\hline $\begin{array}{l}\text { Para enseñar a resolver problemas me da la sensación que } \\
\text { tengo que enseñar a dominar el estado de ánimo de cada } \\
\text { alumno }\end{array}$ \\
\hline
\end{tabular}

\begin{tabular}{c|l|l|l|l|l}
\multicolumn{3}{c}{ Poco } & \multicolumn{5}{c}{ Sobretodo } \\
\hline & $\mathbf{1}$ & $\mathbf{2}$ & $\mathbf{3}$ & $\mathbf{4}$ & \\
\hline & $\mathbf{1}$ & $\mathbf{2}$ & $\mathbf{3}$ & $\mathbf{4}$ & \\
\cline { 2 - 6 } & $\mathbf{1}$ & $\mathbf{2}$ & $\mathbf{3}$ & $\mathbf{4}$ & \\
\cline { 3 - 6 } & $\mathbf{1}$ & $\mathbf{2}$ & $\mathbf{3}$ & $\mathbf{4}$ & \\
\end{tabular}

Figura 3 - Ejemplos de ítems del tercer bloque de preguntas: en qué aspectos de la RP centran la atención.

En el primer y segundo bloque se hace una doble pregunta por cada enunciado propuesto (Figuras 1 y 2). La primera pregunta cuestiona sobre lo que se hace, si evalúa o no lo indicado en el ítem, mientras que la segunda busca identificar lo que se piensa acerca de tener en cuenta cada uno de los aspectos enunciados, de forma que los docentes deben indicar su nivel de acuerdo a través de una escala tipo Likert de 0 a 4 donde 0 indica nada de acuerdo y 4 тиу de acuerdo. Esta pregunta se debe responder de manera independiente a lo que se hace. Sin embargo, en el tercer bloque de preguntas (Figura 3) se responde conforme a una escala tipo Likert de 1 a 4 , donde 1 indica poco y 4 sobretodo.

Cada una de las preguntas analizadas se encuadra en tres categorías de análisis: aspectos del dominio cognitivo/conceptual, aspectos del dominio cognitivo/heurístico y aspectos del dominio afectivo. La diferencia entre lo conceptual y lo heurístico viene dado a que los primeros están ligados a los contenidos matemáticos, mientras que los otros no. 


\subsection{Análisis estadísticos realizados}

Partimos del análisis descriptivo sobre lo que los docentes consideran necesario en la enseñanza de la RP y lo que piensan y evalúan de este contenido, presentando análisis independientes y comparativos. Así, comentamos los resultados sobre la enseñanza de la RP, mostramos los porcentajes de los docentes que consideran importante evaluar algunos de los aspectos descritos en el bloque de preguntas 1 y 2 y destacamos el porcentaje de docentes que manifiesta evaluar dichos aspectos. Ello lo haremos a partir de las categorías de análisis planteadas e iremos comparando los porcentajes de estos tres bloques de preguntas para dar lugar a otros resultados.

A través de la prueba Chi-cuadrado con corrección de Yates y otros estadísticos determinaremos si existe asociación estadística significativa entre el nivel de importancia que los docentes otorgan a evaluar cada uno de los aspectos mencionados en los ítems y el hecho de evaluar o no estos aspectos, y cómo se describe esta asociación.

En las gráficas se distinguen las tres categorías de análisis a partir de colores que hay en las barras. El color negro representa los aspectos del dominio cognitivo/conceptual, el color gris los aspectos del dominio cognitivo/heurístico y el color blanco el dominio afectivo.

\section{Resultados y discusión}

\subsection{Análisis estadístico descriptivo}

A partir del cuestionario CERP se extrae que los profesores de matemáticas ponen de manifiesto la necesidad de enseñar aspectos del dominio cognitivo sobre los del dominio afectivo, haciendo mayor énfasis sobre los aspectos que refieren a la enseñanza de heurísticos que sobre los aspectos que implican algún contenido matemático.

Así, en la Figura 4 se puede observar que la mayoría de los profesores, más del 90\%, manifiestan que consideran muy importante enseñar a sus alumnos a ser intuitivos, a usar el sentido común y enseñarles diferentes estrategias heurísticas para mejorar su capacidad como resolutores de problemas. Mientras que un $68 \%$ considera que los estudiantes serán capaces de resolver problemas si se les enseñan muchas matemáticas. Tan sólo un 33\% de los profesores considera que es muy necesario enseñar a los alumnos a controlar su estado de ánimo al resolver problemas. 


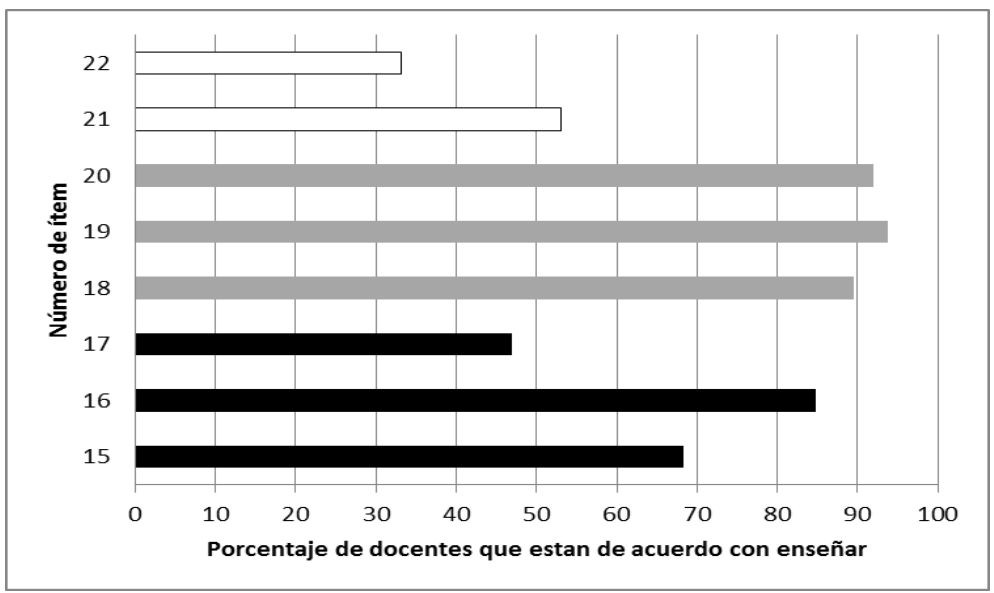

Figura 4 - Porcentaje de docentes que manifiestan estar muy de acuerdo con enseñar os aspectos descritos en cada ítem y que están vinculados con la RP

Estos resultados ponen en evidencia que la enseñanza de la RP no se encuentra limitada por los contenidos matemáticos conceptuales, sino que requiere de una apropiación de contenidos procedimentales diferentes a los algorítmicos. Además, se aprecia una carencia en las ideas que se tienen en torno a la consideración del dominio afectivo a pesar de los numerosos estudios que señalan la influencia de los factores afectivos en la RP (CABALLERO; BLANCO; GUERRERO, 2011; GIL; BLANCO; GUERRERO, 2006; VILA; CALLEJO, 2004).

En la RP un $85 \%$ manifiesta que es muy importante que sus alumnos utilicen las cosas que se les acaban de enseñar y un $45 \%$ que lleguen al resultado exacto. Esto deja como indicio que para los profesores sí es importante el uso de los contenidos matemáticos en la resolución del problema, pero no el hacer los cálculos de manera correcta.

Un $52 \%$ afirma que concede importancia a que un alumno no se bloquee ante la resolución de un problema, lo cual vislumbra que algunos de los docentes no consideran que exista relación entre el estado del ánimo y los bloqueos al resolver problemas. Es decir, no creen necesario integrar aspectos cognitivos y afectivos en la RP tal como nos indican el MEN (1998, 2006) y Blanco, Guerrero y Caballero (2013).

Por otra parte, a través del cuestionario CERP, hallamos que, entre los profesores de matemáticas de secundaria, existe una mayor tendencia a pensar que es más importante el evaluar aquellos aspectos que implican algún contenido matemático. Por otra parte, las tendencias a otorgar un alto nivel de importancia a evaluar los aspectos referentes al uso de heurísticos y los relativos al dominio afectivo, son similares.

Ello se puede observar a través de la Figura 5, donde más del 90\% considera que en la RP es muy importante evaluar la aplicación de métodos matemáticos trabajados en clase y la validación de los resultados a través del proceso matemático empleado. Un $86 \%$ le da un alto 
nivel de importancia a evaluar el hacer cálculos de rutina. El MEN (2006), al hablar del proceso general de la formulación, comparación y ejercitación de procedimientos, hace hincapié en el desarrollo de estos procesos al mencionar que son clave en el mecanismo cognitivo llamado automatización el cual describe de la siguiente manera:

\begin{abstract}
Otro mecanismo cognitivo clave es la automatización, que requiere de la práctica repetida para lograr una rápida, segura y efectiva ejecución de los procedimientos; esta automatización no contribuye directamente al desarrollo significativo y comprensivo del conocimiento, pero sí contribuye a adquirir destrezas en la ejecución fácil y rápida de cierto tipo de tareas. Estas destrezas dan seguridad al alumno y pueden afianzar y profundizar el dominio de dichos conocimientos, pero también pueden perder utilidad en la medida en que se disponga de ayudas tecnológicas que ejecuten dichas tareas más rápida y confiablemente (MEN, 2006, p. $55)$.
\end{abstract}

El porcentaje de profesores que considera muy importante evaluar los aspectos cognitivo/heurísticos, tales como establecer relaciones, buscar y crear diferentes estrategias de resolución, validar modelos y determinar si la respuesta requiere un dato exacto o aproximado, varía entre el $73 \%$ y el $88 \%$. Cabe subrayar que estos porcentajes son menores que los hallados en relación al dominio cognitivo/conceptual.

Los ítems del 10 al 14 hacen mención a aspectos del dominio afectivo que consideran importante evaluar (Figura 5). Concretamente, un $72 \%$ considera importante evaluar la confianza que tiene el alumno sobre sí mismo al hacer frente a situaciones problema y un 69\% el uso de las matemáticas al matematizar un problema. Un 75\% manifiesta que es importante valorar lo que los alumnos piensan de su capacidad para resolver problemas y un $82 \%$ y un 87\% la visión que tienen de las matemáticas y de sí mismo en la RP, respectivamente.

Por otra parte, existe una mayor tendencia a evaluar aquellos aspectos del dominio afectivo relativos a la confianza que tienen los alumnos sobre sí mismos para hacer frente a situaciones problema y su confianza en el uso de las matemáticas al matematizar un problema que a evaluar la visión que tienen los alumnos de las matemáticas en la RP y sobre sí mismos ante dicha actividad. Este resultado cobra importancia si tenemos en cuenta que la autoestima, así como el autoconcepto, son predictores del rendimiento matemático, tanto en el desarrollo de tareas familiares como no familiares, y se encuentran vinculadas al sistema de creencias de los estudiantes (GIL; BLANCO; GUERRERO, 2006). Esta relación que aparece abundantemente en las publicaciones entre la RP y la autoconfianza y la visión que los alumnos tienen de la RP no parece, por tanto, estar asumida por los profesores. 


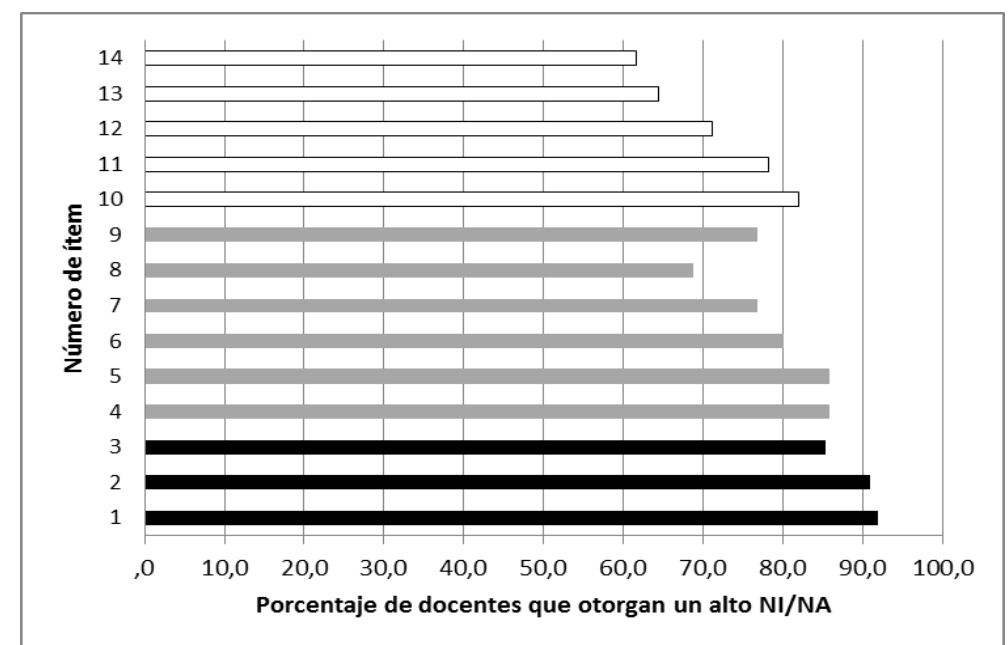

Figura 5- Porcentaje de docentes que manifiesta que es importante evaluar los aspectos de la RP descritos en cada ítem

Finalmente, destacar que los profesores de matemáticas muestran una fuerte tendencia a evaluar los aspectos del dominio cognitivo sobre los del afectivo y hacen un mayor énfasis sobre los aspectos que implican algún contenido matemático que a los referentes al uso de los heurísticos. Ello se puede apreciar en la Figura 6.

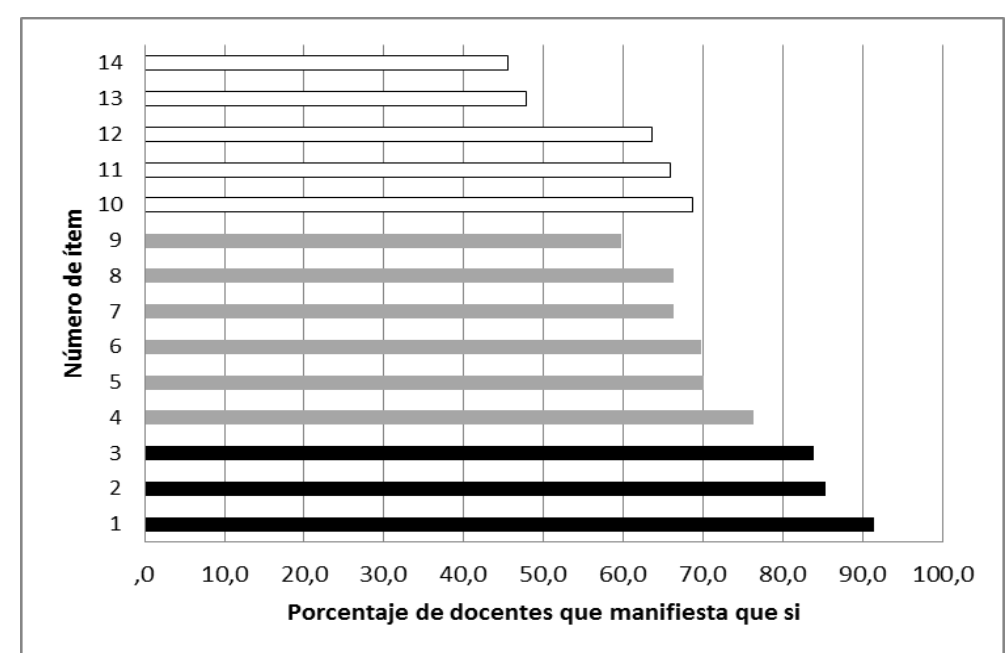

Figura 6 - Porcentaje de docentes que manifiestan evaluar los aspectos de la RP mencionados en cada ítem

Hay una alta tendencia, por parte de los profesores, a manifestar que evalúan los diferentes aspectos que menciona el currículo sobre la RP. Sin embargo, al comparar los porcentajes de las Figuras 5 y 6, es posible determinar que es mayor el porcentaje de docentes que piensan que es importante evaluar los heurísticos o aspectos del dominio afectivo que el porcentaje de docentes que afirma evaluarlos.

Además, los aspectos que están ligados a algún contenido matemático son aquellos que tienen una mayor tendencia a ser evaluados. Encontramos que hay un $15 \%$ de diferencia entre el porcentaje de docentes que manifiesta que evalúa los aspectos que no requieren del uso de la creatividad (ítems del 1 al 3), respecto a los que sí implican su uso (ítems 5 y 6) en 
detrimento de éstos últimos. Los primeros son evaluados por más de un $87 \%$ mientras que los segundos por menos de un 74\%, como se puede apreciar en la Figura 6.

Entre los indicadores de evaluación que se mencionan en los lineamientos curriculares, se hace referencia a los indicadores de comportamientos creativos, donde se otorga importancia a que los estudiantes lleguen al uso de procedimientos no esperados donde se descubren relaciones matemáticas o que ellos sean capaces de ver el problema, lo que significa integrar, ver, asociar donde otros no han visto. Sin embargo la solución no se ve como un acto creativo como tal, ya que ésta puede ser producto del uso de habilidades técnicas (MEN, 1998).

Asimismo, en la Figura 6, es posible observar que el porcentaje de docentes que manifiestan evaluar los aspectos del dominio afectivo varía entre el $48 \%$ y el $72 \%$. La diferencia entre el porcentaje de docentes que manifiesta evaluar estos aspectos, respecto a los que manifiestan darle un alto nivel de importancia es de un $16 \%$ a un $20 \%$, en detrimento de los segundos. Esto podría significar que los profesores reconocen que los factores afectivos inciden en la enseñanza y deberían ser evaluados, pero tienen dificultades para su evaluación.

En general, nos parece importante destacar que se observan claras diferencias en relación a lo que los profesores manifiestan como necesario enseñar, lo que es importante evaluar y lo que dicen que evalúan de la RP. Presentamos a continuación dichas diferencias por categorías de análisis:

En cuanto a los aspectos del dominio cognitivo se encuentran diferencias entre lo conceptual y lo heurístico. En los aspectos del dominio cognitivo/conceptual existe una mayor tendencia a manifestar que es importante evaluarlos y que efectivamente los evalúan, siendo mucho menor la tendencia a manifestar que es necesario enseñar estos aspectos en la RP. Mientras que en lo que a los aspectos del dominio cognitivo/heurísticos respecta, se halla una mayor tendencia a considerar importante la enseñanza de estos aspectos, siendo menor la tendencia referente a la importancia de evaluarlos y aún menor la evaluación de los mismos.

El análisis de los documentos entregados por los profesores (exámenes, talleres etc.) y que no forma parte de este artículo aunque sí de la investigación general, muestra que su repercusión en el aula es aún menor.

Por tanto, los porcentajes de los docentes que manifiestan enseñar los aspectos cognitivo/conceptuales son menores a los que manifiestan evaluarlos. Los porcentajes de profesores que dan una alta importancia a los aspectos relacionados con la enseñanza de los heurísticos son mayores que los que resultan al manifestar que los evalúan. Ésto deja al descubierto que, aunque se asuma que para aprender a resolver problemas hay que ir más allá 
de la realización de procedimientos mecánicos, aún se tiende a rebajar las matemáticas por debajo del nivel de un "libro de cocina" (POLYA, 1986, p.163), donde se limita la evaluación de la RP a comprobar la adquisición del contenido o procesos matemáticos (CONTRERAS; CARRILLO, 2000).

Esto viene sucediendo, a pesar que desde hace varias décadas se promueve el abandono de la práctica tradicional de resumir los resultados matemáticos deseados en forma de destrezas, conceptos y aplicaciones, pidiendo que se evalúen propósitos más generales de la RP y de la comunicación (VILA; CALLEJO, 2004). Además, la evaluación de la RP como contenido viene instaurado en los currículos actuales desde hace varios años, aspecto que no se refleja en la evaluación en matemáticas (CONTRERAS; CARRILLO, 2000; VILA; CALLEJO, 2004).

En relación a los aspectos del dominio afectivo se visualiza una menor tendencia a manifestar la necesidad de enseñar a manejar estos aspectos, aunque esta tendencia se incrementa al referir que se evalúan, y aumenta aún más cuando se manifiesta que se considera importante llegar a evaluarlos.

En la enseñanza consideramos que los resultados son reflejo de una respuesta dada sobre aspectos que los profesores consideran que no tienen repercusión en el aprendizaje de las matemáticas, en este caso, el estado de ánimo. Diversas investigaciones que refieren a la evaluación en matemáticas indican que, al evaluar el dominio afectivo, la evaluación únicamente se centra en el comportamiento de los estudiantes, su responsabilidad y la honestidad al no hacer copia en los exámenes (MORENO; ORTIZ, 2008).

No obstante, en el presente estudio hemos podido constatar que los profesores aunque consideran importante la visión que tienen los estudiantes de sí mismos ante la RP, su capacidad y confianza como resolutores, su autoconcepto o la atribución causal del éxito y fracaso escolar en el rendimiento de sus alumnos, no son conscientes de la repercusión de aspectos del dominio afectivo en el rendimiento de los estudiantes, siendo aspectos que se investigan por diversos autores como Gómez-Chacon (2000) y Blanco, Guerrero y Caballero (2013).

Por tanto, es posible continuar afirmando que el aprendizaje se viene midiendo por los logros académicos de los aspectos cognitivos, a pesar de que se reconozca que los aspectos del dominio afectivo también determinan la calidad del aprendizaje.

\subsection{Análisis estadístico inferencial de la distribución conjunta}


Tal como se aprecia en la Figura 7, en los ítems del 1 al 14 se encuentran cuatro tipos de respuesta: los que dicen que sí evalúan y dan un alto Nivel de Importancia (NI), los que evalúan y dan un bajo NI; los que no evalúan y le dan un bajo NI, y los que no evalúan y dan un alto NI. Consideramos interesante verificar si existe algún tipo de asociación estadística que describa las respuestas que dan los profesores a estas dos preguntas, para ello aplicamos las pruebas Chi-cuadrado, Chi-cuadrado con la corrección de Yates y el estadístico exacto de Fisher sobre las tablas de frecuencia.

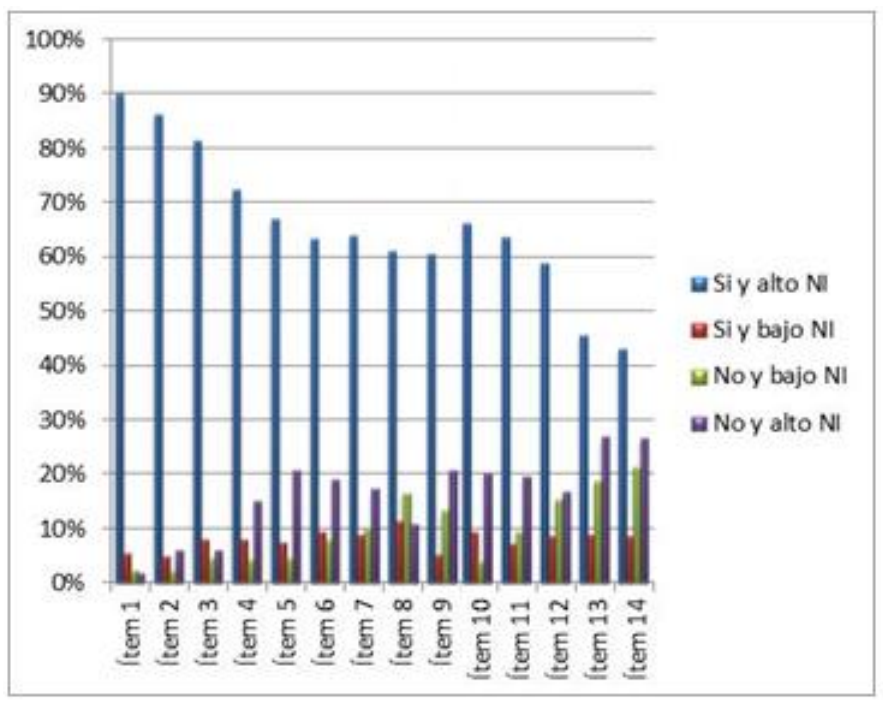

Figura 7- Afirmaciones hechas al indicar si evalúa o tiene en cuenta (si, no) y nivel de importancia o acuerdo (NI)

En los ítems del 1 al 4, solo tenemos en cuenta el p-valor del estadístico exacto de Fisher, dado que más de un $20 \%$ de las casillas de las tablas de contingencia presentan una frecuencia esperada menor a 5 . En el resto de ítems usamos el contraste de las tres pruebas.

De este modo, en la Tabla 1 observamos que los ítems 5 y 10 presentan un $p$-valor $>.05$ en las tres pruebas (Tabla 1), y el ítem 4 en el estadístico exacto de Fisher encontramos que su $p$-valor $>.05$ en la significación exacta bilateral. Es por ello que indicamos que en estos ítems no hay una asociación estadística significativa entre las manifestaciones que realizan los profesores sobre lo que hacen y lo que piensan de la evaluación de la RP. Aceptamos que el nivel de importancia que otorgan los docentes a evaluar el que los estudiantes hagan pronósticos sobre la solución del problema, el uso de diversos lenguajes para expresar ideas matemáticas pertinentes a partir de situaciones problema y el estructurar argumentos sobre las acciones desarrolladas en la RP, es independiente de si lo evalúan o no. 
Tabla 1 - Pruebas de asociación estadística entre las variables hacen y piensan.

Y medidas de intensidad de asociación.

\begin{tabular}{|c|c|c|c|c|c|c|c|c|}
\hline & chi-cu & ladrado & $\begin{array}{r}\text { chi-cua } \\
\text { corre } \\
\mathrm{Y}\end{array}$ & $\begin{array}{l}\text { drado con } \\
\text { ción de } \\
\text { ates }\end{array}$ & $\begin{array}{l}\text { est. e } \\
\text { de Fis }\end{array}$ & & & \\
\hline & Valor & $\begin{array}{l}\text { Sig. } \\
\text { asintótica } \\
\text { (bilateral) }\end{array}$ & Valor & $\begin{array}{l}\text { Sig. } \\
\text { asintótica } \\
\text { (bilateral) }\end{array}$ & $\begin{array}{r}\text { Sig. } \\
\text { exacta } \\
\text { (bilateral) }\end{array}$ & Phi & $\begin{array}{r}\text { sig. } \\
\text { aproximada }\end{array}$ & $\begin{array}{l}\text { Residuos } \\
\text { tipificados } \\
\text { corregidos }\end{array}$ \\
\hline Ítem 1 & $29,306^{\mathrm{a}}$ & 0,000 & 22,869 & 0,000 & 0,000 & 0,381 & 0,000 & 5,41 \\
\hline Ítem 2 & $12,567^{\mathrm{a}}$ & 0,000 & 9,404 & 0,002 & 0,005 & 0,253 & 0,000 & 3,54 \\
\hline Ítem 3 & $19,461^{\mathrm{a}}$ & 0,000 & 16,516 & 0,000 & 0,000 & 0,314 & 0,000 & 4,41 \\
\hline Ítem $4 *$ & $4,882^{\mathrm{a}}$ & 0,027 & 3,764 & $0,052 *$ & $\mathbf{0 , 0 5 5 *}$ & 0,157 & 0,027 & 2,21 \\
\hline Ítem $5^{*}$ & 2,170 & $0,141 *$ & 1,495 & $0,221 *$ & $0,208 *$ & 0,105 & 0,141 & 1,47 \\
\hline Ítem 6 & 7,538 & 0,006 & 6,431 & 0,011 & 0,011 & 0,194 & 0,006 & 2,75 \\
\hline Ítem 7 & 14,619 & 0,000 & 13,073 & 0,000 & 0,000 & 0,277 & 0,000 & 3,82 \\
\hline Ítem 8 & 38,058 & 0,000 & 35,874 & 0,000 & 0,000 & 0,444 & 0,000 & 6,17 \\
\hline Ítem 9 & 28,134 & 0,000 & 26,107 & 0,000 & 0,000 & 0,383 & 0,000 & 5,30 \\
\hline Ítem 10* & 0,644 & $0,422 *$ & 0,310 & $0,578 *$ & $0,464 *$ & 0,058 & 0,422 & 0,80 \\
\hline Ítem 11 & 13,814 & 0,000 & 12,275 & 0,000 & 0,000 & 0,268 & 0,000 & 3,72 \\
\hline Ítem 12 & 28,136 & 0,000 & 26,269 & 0,000 & 0,000 & 0,380 & 0,000 & 5,30 \\
\hline Ítem 13 & 13,939 & 0,000 & 12,722 & 0,000 & 0,000 & 0,277 & 0,000 & 3,73 \\
\hline Ítem 14 & 16,406 & 0,000 & 15,125 & 0,000 & 0,000 & 0,299 & 0,000 & 4,05 \\
\hline
\end{tabular}

En el resto de los ítems observamos que el p-valor <.05 en las correspondientes pruebas aplicadas. Consecuentemente, existe una asociación estadística significativa entre las manifestaciones que hacen los docentes sobre lo que hacen y piensan de la evaluación de la $\mathrm{RP}$, por lo que se asume que las respuestas dadas no han sido atribuidas al azar, sino que cabe esperar que para producirse esta diferencia es probable que haya operado una decisión y una voluntad en indicar el nivel de importancia dependiendo de si lo evalúa o no.

Esto puede ser causa de la reflexión personal del docente ante sus prácticas de evaluación o ser acusado a que las respuestas las diera siguiendo el principio de deseabilidad social más allá del azar. No obstante, la intensidad de esta asociación es baja en el ítem 6 y moderadamente baja en el resto de ítems. Describimos esta asociación de la siguiente forma: el nivel de importancia que otorgan los docentes depende de si han manifestado que evalúan o no dicho aspecto, otorgando un alto nivel de importancia a aquellas cuestiones que manifiestan evaluar, y un bajo nivel de importancia a las que no. Ésta es la asociación lógica a discurrir pero, como observamos, no siempre se da esta relación hasta el punto que en la mayoría de los ítems hay docentes que afirman no evaluar algunos aspectos y sin embargo le dan un alto nivel de importancia.

El mayor porcentaje de estos casos se observa en aspectos del dominio afectivo, luego en los aspectos cognitivos/heurísticos, después en el tipo de actividades que se proponen y por último en los aspectos relacionados con conceptos y contenidos matemáticos. Así, el 59.04\% de los docentes que dicen no evaluar (26.92\% de la población total) el ítem 14 (¿Evalúo la 
visión que cada estudiante tiene de sí mismo en la RP?) le dan un alto nivel de importancia. En el ítem 2 (¿Evalúo el validar los resultados a través del proceso matemático empleado?), el $70.59 \%$ de los que manifiestan no evaluar $(6.09 \%$ del total) y darle un alto nivel de importancia. Igual sucede con otros ítems como en el 9 (¿Evalúo el ajustar o modificar el plan implementado para la resolución del problema en caso de ser necesario?).

\section{Conclusiones}

El trabajo muestra que existen aspectos relacionados con la RP que los docentes consideran que son importantes enseñar y aprender y, por tanto, objetos de evaluación, pero que no siempre los evalúan. En contraposición, también se encuentran otros aspectos que los docentes manifiestan darles menos importancia en la enseñanza/aprendizaje y sin embargo son los aspectos que más se evalúan.

Por otra parte, los resultados permiten concluir que en la RP se continúa priorizando, en la acción, la evaluación de aspectos que refieren al dominio cognitivo sobre los del afectivo. Y, aunque en la enseñanza de los aspectos que se enuncian del dominio afectivo no se consideran importantes, se visualiza que hay un mayor nivel de conciencia por parte de los docentes en la necesidad de tener en cuenta los aspectos del dominio afectivo en la evaluación. Además, dentro del dominio cognitivo observamos que se hace un menor énfasis en la evaluación de las estrategias heurísticas que sobre los aspectos propios al conocimiento matemático, a pesar de que se ponga de manifiesto que su enseñanza y evaluación es más importante.

Al asumir que la evaluación es más que un proceso para emitir un juicio/calificación final y que se integra en el proceso de enseñanza-aprendizaje, consideramos que esta integración debe ser visible, también, cuando se habla de la RP en matemáticas. De tal modo que, en la evaluación en matemáticas en general y de la RP en particular, no sólo se enfatice la evaluación de los aspectos cognitivo conceptuales, sino que también se tengan en cuenta la evaluación de los aspectos cognitivos/heurísticos y afectivos, tal como sugieren las propuestas curriculares.

A la vez, que si reconocemos que a través de la evaluación se identifican las dificultades que presentan los estudiantes en torno a los aspectos cognitivo/heurísticos y afectivos y se generan estrategias para suplir las necesidades observadas, se puede contribuir a que los estudiantes lleguen a ejercer control sobre ellos mismos, haciendo posible tanto la autoevaluación como la metacognición, de modo que se optimicen los resultados ante la RP. 
Los diferentes resultados de la investigación nos indican la necesidad de continuar profundizando sobre las concepciones y prácticas de los docentes de matemáticas de secundaria en torno a la evaluación de la RP. Así como el integrar en la formación de futuros profesores de matemáticas referencias prácticas y teóricas sobre el dominio afectivo en la enseñanza de las matemáticas y la evaluación de heurísticos.

\section{Referencias}

ÁLVAREZ, R.; BLANCO, L. J. Sobre la evaluación en Matemáticas en Secundaria. SUMA, Badalona, n. 76, p. 47-54, 2014.

ACEVEDO, M.; PÉREZ, M.; MONTAÑEZ, J.; HUERTAS, C.; VEGA, G. Propuesta para la actualización teórica de las pruebas saber y de estado (2004-2005). Bogotá: Universidad Nacional de Colombia, v. 1, 2005. 123 p.

BLANCO, L. J.; BARRANTES, M. Concepciones de los estudiantes para maestro en España sobe la geometría escolar y su enseñanza-aprendizaje. RELIME, México, v. 6, n. 2, p. 107-132, 2003.

BLANCO, L.J.; GUERRERO, E.; CABALLERO, A. Cognition and Affect in Mathematics Problem Solving with Prospective Teachers. The Mathematics Enthusiast, Montana, v. 10, n. 1 y 2, p. 335$364,2013$.

BROWN, G. T. L.; REMESAL, A. Prospective teachers' conceptions of assessment: a cross-cultural comparison. The Spanish Journal of Psychology, Madrid, n. 15, p. 75-89, 2012.

CABALLERO, A.; BLANCO, L. J.; GUERRERO, E. Problem solving and emotional education in initial primary teacher education. Eurasia Journal of Mathematics, Science \& Technology Education, Ankara, v. 7, n. 4, p. 281-292, 2011.

CÁRDENAS, J. A. La evaluación de la Resolución de Problemas en Matemáticas: concepciones y prácticas de los profesores de secundaria. 2014. 453 p. Tesis (Doctorado en Investigación de la Enseñanza-Aprendizaje de las Ciencias Experimentales, Sociales y de las Matemáticas) - Facultad de Ecuación, Universidad de Extremadura, Badajoz, 2014.

CASTRO, E. Resolución de problemas. Ideas, tendencias e influencias en España. En: LUENGO, R.; GÓMEZ, B.; CAMACHO, M.; BLANCO, L. J. Investigación en educación matemática XII. Badajoz: SEIEM, 2008. p. 93-111.

CASTRO, H.; MARTÍNEZ, R.; FIGUEROA, Y. Fundamentaciones y orientaciones para la implementación del decreto 1290 del 16 de abril del 2009. Evaluación del aprendizaje y promoción de los estudiantes en los niveles de educación básica y media. Bogotá: MEN, 2009. 108 p. Disponible en: 〈http://www.mineducacion.gov.co/1621/articles-213769 archivo pdf evaluacion.pdf >. Acceso en: 19 may. 2011.

CONTRERAS, L. C.; CARRILLO, J. Resolución de problemas en los Albores del Siglo XXI: Una visión Internacional desde Múltiples Perspectivas y Niveles Educativos. Huelva: Hergué, Editora Andaluza, 2000. $261 \mathrm{p}$.

GAIRÍN, J. M.; MUÑOZ, J. M.; OLLER, A. M. Propuesta de un modelo para la calificación de exámenes de matemáticas. En: ESTEPA, A.; CONTRERAS, J.; DEULOFEU, M. C.; PENALVA, F. 


\section{J.; GARCÍA, L.; ORDÓNEZ (Coord.). Investigación en Educación Matemática XVI. Baeza:} SEIEM, 2012. p. 261-274.

GIL, N.; BLANCO, L. J.; GUERRERO, E. El papel de la afectividad en la Resolución de Problemas. Revista de Educación, Madrid, n. 340, p. 551-569, 2006. Disponible en: < http://www .revistaeducacion.mec.es/re340/re340_20.pdf >. Acceso en: 12 may. 2014.

GIMÉNEZ, J. Evaluación en matemáticas. Una integración de perspectivas. Madrid: Síntesis, 1997. $336 \mathrm{p}$.

GODOY, L. Evaluación en Matemáticas: Análisis de exámenes de Geometría en $3^{\circ}$ de ESO. 2013. 159 p. Dissertação (Mestrado em Investigación de la Enseñanza-Aprendizaje de las Ciencias Experimentales, Sociales y de las Matemáticas) - Facultad de Educación, Universidad de Extremadura, Badajoz, 2013.

GÓMEZ-CHACON, M.I. Matemática emocional. Los afectos en el aprendizaje matemático. Madrid: Narcea, 2000, 280 p.

GOÑI, J. La evaluación de las competencias determinará el currículo de matemáticas. En: GOÑI, J. 32-2 ideas clave. El desarrollo de la competencia matemática. España: GRAO, 2008. p. 167-185.

GOÑI, J. M. Las finalidades del currículo de matemáticas en secundaria y bachillerato. In J. M. GOÑI (Ed.), Didáctica de las Matemáticas. Barcelona: Grao, 2011, p. 9-25.

HARLEN, W. The role of assessment in developing motivation for learning. In: GARDNER, J. (Ed.). Assessment and Learning. California: Sage, 2012. p. 171-183.

HIDALGO, S.; MAROTO, A.; ORTEGA, T.; PALACIOS, A. Influencia del Dominio Afectivo en el Aprendizaje de las Matemáticas. En: MELLADO, V.; BLANCO, L. J.; BORRACHERO, A. B.; CÁRDENAS, J. A. Las Emociones en la Enseñanza y Aprendizaje de las Ciencias y las Matemáticas. Badajoz: Grupo DEPROFE, 2013. p. 217-243.

MINISTERIO DE EDUCACIÓN NACIONAL - MEN. Lineamientos curriculares para matemáticas. Serie lineamientos curriculares. Bogotá: Ministerio de Educación Nacional, 1998, Colombia. 103 p.

MINISTERIO DE EDUCACIÓN NACIONAL - MEN. Estándares curriculares en Matemáticas. Bogotá: Ministerio de Educación Nacional, 2006.

MENGUAL, E.; GORGORIÓ, N.; ALBARRACÍN, L. Validación de un instrumento para la calificación de exámenes de Matemáticas. En: BERCIANO, A.; GUTIÉRREZ, G.; ESTEPA, A.; CLIMENT, N. (Coord.). Investigación en educación matemática XVII. Bilbao: SEIEM, 2013. p. 367-381.

MORENO, I.; ORTIZ, J. Docentes de Educación Básica y sus concepciones acerca de la evaluación en matemáticas. Revista Iberoamericana de Evaluación Educativa, México, v. 1, n. 1, p. 140-154. 2008. Disponible en: <http://www.rinace.net/riee/numeros/vol1-num1/art10.pdf >. Acceso en: 21 oct. 2013.

MULLIS, I. V. S.; MARTIN, M. O.; FOY, P.; ARORA, A. TIMSS 2011 International Results in Mathematics. Chestnut Hill: TIMSS \& PIRLS International Study Center, Boston College, 2012. 520 p. 
ORGANIZACIÓN PARA LA COOPERACIÓN Y EL DESARROLLO ECONÓMICOS - OECD. PISA 2012 Results: Creative Problem Solving: Students' Skills in Tackling RealLife Problems, OECD Publishing, 2014.

PINO, J.; BLANCO, L. J. Análisis de los problemas de los libros de texto de Matemáticas para alumnos de 12 a 14 años de edad de España y de Chile en relación con los contenidos de proporcionalidad. Publicaciones, Santiago de Chile, n. 38, p. 63-88, 2008.

POLYA, G. Cómo plantear y resolver problemas. México: Trillas, 1986. 217 p.

PUIG, L. Resolución de Problemas: 30 años después. En: LUENGO, R.; GÓMEZ, B.; CAMACHO, M.; BLANCO, L. J. Investigación en educación matemática XII. Badajoz: SEIEM, 2008. p. 93-111.

SANTOS-TRIGO, L. M. La resolución de problemas matemáticos. Fundamentos cognitivos. México: Trillas, 2007. 254 p.

SANTOS-TRIGO, L. M. La resolución de problemas matemáticos: Avances y perspectivas en la construcción de una agenda de investigación y práctica. En: LUENGO, R.; GÓMEZ, B.; CAMACHO, M.; BLANCO, L. J. Investigación en educación matemática XII. Badajoz: SEIEM, 2008. p. 93-111.

SCHOENFELD, A. Problem solving in the United States, 1970-2008: research and theory, practice and politics. ZDM The International Journal on Mathematics Education, Hamburg, v. 39, n. 5 y 6 , p. $537-551,2007$.

VILA, A.; CALLEJO, M. L. Matemáticas para aprender a pensar. El papel de las creencias en la resolución de problemas. Madrid: Narcea, 2004. 218 p.

Submetido em Janeiro de 2015. Aprovado em Outubro de 2015. 


\section{Anexo 1 - Ítems del CERP empleados para las categorías contempladas en este artículo:}

1 ¿Evalúo el aplicar métodos matemáticos trabajados en clase en la RP?

2 ¿Evalúo el validar los resultados a través del proceso matemático empleado?

3 ¿Evalúo el hacer cálculos de rutina para la solución de problemas?

4 ¿Evalúo el visualizar las relaciones existentes entre los diferentes elementos del problema?

5 ¿Evalúo el usar estrategias o métodos diferentes a los que se han trabajado en clase para la RP?

6 ¿Evalúo el proponer más de una estrategia a seguir de acuerdo con las condiciones del problema?

$7 \quad$ ¿Evalúo el validar el modelo planteado para la solución del problema?

8 ¿Evalúo el reconocer si la respuesta que requiere un problema es un dato exacto o un dato aproximado?

9 ¿Evalúo el ajustar o modificar el plan implementado para la resolución del problema en caso de ser necesario?

10 ¿Evalúo la confianza en el uso de las matemáticas al matematizar un problema?

11 ¿Evalúo el desarrollo de la confianza en sí mismo para hacer frente a situaciones problema?

12 ¿Para conocer cómo van los procesos de RP de los estudiantes, tengo en cuenta lo que ellos piensan de su capacidad de resolver problemas?

13 ¿Evalúo la visión que los estudiantes tienen de las matemáticas en la RP?

14 ¿Evalúo la visión que cada estudiante tiene de sí mismo en la RP?

15 Doy importancia a que mis estudiantes al resolver problemas obtengan el resultado exacto

16 Doy importancia a que mis estudiantes al resolver problemas utilicen las cosas que acabamos de explicar

17 Para enseñar a resolver problemas me da la sensación que tengo que enseñar muchas matemáticas

18 Para enseñar a resolver problemas me da la sensación que tengo que enseñar a ser intuitivo y a utilizar el sentido común

19 Para enseñar a resolver problemas me da la sensación que tengo que enseñar estrategias como por ejemplo hacer esquemas, representaciones,...

20 Para enseñar a resolver problemas me da la sensación que tengo que enseñar estrategias como por ejemplo probar con casos más sencillos, con ejemplos,...

21 Cuando miro cómo resuelve un alumno un problema, doy importancia a que no se quede bloqueado en ningún momento

22 Para enseñar a resolver problemas me da la sensación que tengo que enseñar a dominar el estado de ánimo de cada alumno 